\title{
Critical Care Nurses' Cognitive Ergonomics Related to Medical Device Alarms
}

\author{
Running title: Device Alarms Decision Making
}

Shu-Fen Wung, PhD, RN, ACNP-BC, FAAN, Associate Professor ${ }^{1}$; Marilyn Rose Schatz, DNP, $\mathrm{RN}, \mathrm{ACNP}-\mathrm{BC}^{2}$

The University of Arizona College of Nursing ${ }^{1}$, Tucson, AZ; Pulmonary Consultants of Mesa, Mesa, $A Z^{2}$

Shu-Fen Wung, PhD, RN, ACNP-BC, FAAN

Associate Professor

The University of Arizona College of Nursing

$1305 \mathrm{~N}$ Martin Avenue

Tucson, AZ 85721-0203

520-626-4305

Fax 520-626-4062

wung@arizona.edu

Marilyn Rose Schatz, DNP, RN, ACNP-BC

Nurse Practitioner

Pulmonary Consultants of Mesa

1245 N. Tucana Ct.

Gilbert, AZ 85234

schatz6@me.com

Corresponding author: Dr. Shu-Fen Wung

Disclosure statement: There is no commercial or financial conflicts of interest for all authors. There is no grant or other financial support used for the study. 


\section{Synopsis:}

This study uniquely gained insight into the intricacy of ICU nurses' decision-making process when responding to and managing device alarms. Difficulty in responding to alarms included low staffing, multiple job responsibilities, and competing priority tasks. Novice nurses are more tolerant of alarms sounding due to a lower threshold of comfort with re-setting or silencing alarms while experienced nurses are more comfortable re-setting alarm limits to the patient's baseline. Understanding the decision-making process used by nurses can guide the development of policies and learning experiences that are crucial clinical support for alarm management. 


\section{Key words:}

decision-making, monitor alarms, alarm fatigue, intensive care, human factors

\section{Key points:}

- ICU nurses integrate multiple factors, including tone, duration, and type of the alarm, priority of other competing activities, knowledge of patient acuity, and ICU experience, into considerations when deciding how quickly or even whether an alarm will be responded to.

- Difficulty in responding to alarms included low staffing, multiple job responsibilities, and competing priority tasks.

- Less experienced nurses tend to rely on monitor alarms to alert them to a patient problem and are more tolerant of alarms sounding due to a lower threshold of comfort with re-setting or silencing alarms.

- Future studies on alarm management should include effective use of alarm-related equipment trainings and/or innovative user-centered alarm management clinical decision support systems to enhance intuitive nurse-device interactions. 


\begin{abstract}
Background: Excessive numbers of alarms generated from multiple medical devices in the intensive care unit (ICU) have significant consequences for the nurses and the care they provide and how they use their time and attention. The purpose of this study was to gain insight into the intricacy of ICU nurses' decision-making process related to prioritizing, responding and managing medical device alarms in ICU, when under conditions of high uncertainty, time pressure and risk.

Methods: This was a descriptive study involving interview of 16 ICU nurses. Semi-structured Jopen-ended interview questions were developed based on the Situated Clinical DecisionMaking Framework and were formulated to probe cognitive decision-making processes related to device alarms.

Results: ICU nurses integrate multiple factors into considerations when deciding how quickly or even whether an alarm will be responded to. These included tone, duration, and type of the alarm, priority of other competing activities, adequacy of staffing, knowledge of patient acuity, and years of ICU experience. When responding to an alarm, ICU nurses gather data beyond just the alarm itself. Level of an alarm (e.g., crisis, advisory), presence of a backup warning mechanism, patients' clinical status, and nurses' comfort level affect the decision if an alarm may be disabled. Nurses with less ICU experience were hesitant to disable alarms.

Conclusions: ICU nurses took multiple factors into consideration when deciding how quickly to respond to alarms. They integrate collected information, their clinical experience, and their knowledge of the patient's condition to fit into a deep understanding of the whole picture. Understand such rich decision-making process used by ICU nurses can guide the development of policies and learning support that are crucial for alarm management.
\end{abstract}




\section{INTRODUCTION}

Acute care nurses are responsible for the safety of critically ill patients around the clock in the increasingly complex technology-rich intensive care units (ICUs) by making distinctions between clinical changes that warrant emergent intervention and those that do not. ${ }^{1}$ In this high pressure work environment, numerous devices, often complicated, are commonly used to enable continuous measurements of physiologic function of the patient and function of the medical devices. ${ }^{2}$ To increase safety, alarms are embedded to almost every device in the ICU to alert critical care nurses of potential problems early to institute appropriate interventions. Nurse are expected to monitor and respond appropriately to the plethora of pathophysiologic data and alarms produced by the patient and devices.

Ironically, the very alarm systems created to enhance patient safety have become an urgent patient safety concern. ${ }^{2}$ Just a bedside cardiopulmonary monitor alone generates 187 audible alarms per bed per day, averaging one alarm every 7.7 minutes. ${ }^{3}$ To exacerbate the alarm problem further, up to $90 \%$ of alarms are deemed false or nonactionable with very few indicating serious clinical events. ${ }^{4-10}$ Constant demand to respond to alarms and mistrust of the alarm system due to high numbers of clinically irrelevant alarms reduce alertness of the clinicians. Clinicians become accustomed to ignoring false alarms and, as a result, may overlook an alarm that signals a true emergency, producing a phenomenon known as alarm fatigue. Patient injury and death resulted from inadequate attention to alarms have been reported. ${ }^{11}$ In addition, silencing alarms is the second most common task performed by nurses, accounting for approximately $16 \%$ of a nurse's bedside tasks. ${ }^{12}$

As more tasks are handled by technology, humans are becoming responsible for tasks that require inference, diagnoses, judgement, and decision making. ${ }^{13}$ In the ICU, ever-growing complex technology places nurses at a greater cognitive demand. As defined by the International Ergonomics Association, cognitive ergonomics (or human factors) is "concerned 
with mental processes, such as perception, memory, reasoning, and motor response, as they affect interactions among humans and other elements of a system. (Relevant topics include mental workload, decision-making, skilled performance, human-computer interaction, human reliability, work stress and training as these may relate to human-system design.)"14 Decision making, a process that humans use to determine a forward path of action, ${ }^{15}$ is one of 10 key human factors topics relevant for patient safety. ${ }^{16}$ To design an effective alarm management program, we propose to focus on cognitive ergonomics by understanding critical care nurses' cognitive processes, specifically intricacy of decision-making capabilities, related to prioritizing, responding and managing medical device alarms in ICU, when under conditions of high uncertainty, time pressure and risk.

\section{Theoretical Framework}

The Situated Clinical Decision-Making Framework provides a structured approach to analyze nurses' decision-making in clinical practice and to guide selection of relevant strategies to support development of clinical decision making ${ }^{17}$. In this framework, four phases have been identified to comprise the clinical decision-making process; cues, judgments, decisions, and evaluation of outcomes ${ }^{18}$. A nurse's clinical decision-making process is triggered by recognition of a cue from the patient, either a response or lack of something expected. Once the initial cue is noticed, the nurse collects additional cues to build an understanding of the situation. Cues can be collected from multiple sources, including patient observation and assessment, statements from patients or others, objective data, and the nurse's intuition. Ongoing cue collection is informed by a nurse's evolving understanding of the situation. For example, when a device alarm sounds, the nurse is cued to a possible change in patient condition. This alarm may trigger a reminder of a comment made by the patient, such as a previous complaint of discomfort. Judgment is defined as the best conclusion that can be reached at a point in time, given the available information. This reflects the dynamic process between possible judgements 
and collected cues. Ongoing cue further informs one's judgement. Decision is committing to a course of action, whether it is "waiting and watching" and/or "trying something". ${ }^{18}$ The nurse may choose to proceed with a tentative course of action and remain open to revise the actions as new information becomes available. Evaluation of outcomes is a reflection on the effectiveness of cue collection, judgment and decision, and whether further action is indicated. Reflecting on the outcome is an opportunity for clinical improvement and nurses often unconsciously go over their decisions to assure the best decision has been made for the patient in that particular situation. ${ }^{17}$

\section{METHODS}

\section{Design, Setting and Sample}

This was a descriptive study aimed at understanding the complexities of the decisionmaking process related medical device alarms among ICU nurses. A semi-structured interview was designed and conducted with a sample of 16 registered nurses from a 30-bed combined medical-surgical, cardiac and cardiovascular surgical ICU in a 178-bed community hospital. The nurses typically work 12-hour shifts. Eight nurses, seven women and one man, were interviewed from each shift to obtain equal representation from each timeframe.

Since nurses' decision-making often evolves with experience, ${ }^{17}$ we analyzed decisionmaking process related to medical device alarm by nurses' clinical experience, advanced beginners and experts. Benner ${ }^{19}$ defined an advanced beginner nurse as a person who has experienced actual clinical situations to identify their significant elements and the expert nurse has an instinctive grasp of the situation and can pinpoint the problem without having to consider options that are not useful in a given situation. In this study, advanced beginner and expert were arbitrary defined as having five or less years and five years of ICU experience, respectively.

\section{Instrument}

Semi-structured open-ended interview questions were developed based on decision- 
making process of the Situated Clinical Decision-Making Framework and formulated to probe a situation related to device alarm to bring out the cognitive processes used in making-decision. Questions focused on device alarm identification, alarm management, and parameter settings on the monitor. There were total of 15 questions, including cues initiating the decision-making process, judgment on how nurses processed the cues, decisions made after judging the cues, reflection on areas amenable for improvement, such as training on the monitors or refresher courses on cardiac rhythm interpretation. Content validity of this semi-structured interview was supported by three doctorally prepared nurse researchers and three board-certified acute care nurse practitioners.

\section{Data Collection Protocol}

Upon approval from institutions' Human Subjects Protection Programs and permission from the study ICU, the purpose of this study was presented at a unit meeting and interested participants were encouraged to contact the researcher. Informed consent was obtained from each nurse prior to the interview. Each interview was recorded and later transcribed verbatim to ensure completeness of the data.

\section{Data Analysis}

A content analysis was performed and began with read and reread the transcription several times to obtain an overview of the data. General categorization was based on the four phases of clinical decision-making process: cues, judgments, decisions, and evaluation of outcomes. Further analysis of transcript was performed to identify concepts within each of the four themes and interpret the meaning of responses to better understand decision-making process related to monitor alarms. Descriptive statistics were used to describe demographic data.

\section{RESULTS}

\section{Sample Characteristics}


Sixteen ICU nurses participated in the interview. Of these, $38 \%$ and $62 \%$ were advanced beginner and expert, respectively. The mean age of ICU nurses was $38 \pm 11$ years, ranged from 27 to 60 years old, and the mean years of ICU experience was $9 \pm 7$ years, ranged from 1.5 to 21 years. Educational preparation of these nurses included two with diploma degrees, five with an Associate Degree of Nursing, eight with a Bachelor of Science in Nursing, and one with a Master's of Science in Nursing.

Nurses' cognitive ergonomics, specifically decision-making related to medical device alarms, is presented below, according the four areas of mental processing (cues, judgments, decisions and evaluation of outcomes) of the Situated Clinical Decision-Making Framework.

\section{Cues}

Alarms in the ICU. Nurses indicated that they felt overwhelmed by monitor alarms, yet, the number of hourly alarms they heard or dealt with varied greatly within the same ICU with only $38 \%$ heard more than five alarms per hour in their last shift. Of the nurses who heard more than five alarms per hour, $80 \%$ were expert nurses. An advanced beginner nurse with 1.5 years of ICU experience stated "There is always something, whether it is a ventilator or the actual ICU monitors, like the vital sign machine, the SCD [sequential compression device] machine, or the beds. There are always alarms going off every minute or so.”

Cues Affect Timeliness of Alarm Response. Disregard years of ICU experience, among nurses interviewed, there was an unified initial response to an alarm - "It needs attention." ICU nurses took multiple factors into consideration when deciding how quickly to respond to alarms. These included the tone of the alarm, priority of other competing activities, and patient status. Factor that determines how fast or even whether an alarm will be responded to often depends more on the volume of the alarm bell than the urgency of the underlying condition. Thirty-three percent of advanced beginner nurses stated that they might ignore the alarm when they are engaged in a higher priority task. "If I am in the middle of an emergent situation, I probably shut 
a lot of them [alarms] out", "If I happen to be doing something really really important, I'll let it [alarm] slide." Similarly, expert nurses stated that their timeliness of response [to an alarm] was determined by the tone of the alarm as well as the patient status. "Well I think all alarms are important, so the high level alerts you always want to check immediately, but I like to get to my alarms as soon as possible". "Any sound you have to do it quickly, because it is alarming for a reason."

\section{Judgement}

Judgement on Alarm Urgency. Nurses interviewed judged the urgency of an alarm based on the tones of the alarm, knowledge of the patient's condition as well as immediate visualization of the patient. The urgency of an alarm is judged "by the tones of the alarm and my knowledge of the patient's condition", an advanced beginner responded. An expert nurse stated, "Is it a low level alarm? Are my alarm parameters correct? If you have a patient who has been in sinus rhythm [and the heart rates are] in the 80s, and all of a sudden they're in atrial fib [fibrillation] with a [heart] rate of 120, well that needs some pretty direct attention.

Judgement on Alarm Validity. Nurses gathered information through both visual and auditory means to decide if an alarm is authentic. An advanced beginner stated, "if it's a real alarm, as opposed to artifact, l'd like to look at the patient to see if there are any safety concerns." Responses from experts regarding ways to authenticate an alarm included: "It depends on the alarm. If you look up and see your EKG [electrocardiographic] monitor, does it look like a Vtach [ventricular tachycardia] or Vfib [ventricular fibrillation]? If the patient has an art [arterial] line, do I have a pressure? I correlate those two. If it [EKG monitor] says Vtach and you have a good arterial pressure, then it is probably not Vtach."

\section{Decision}

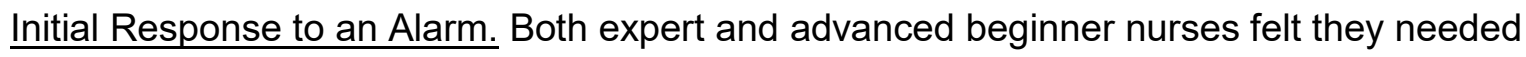
to observe the patient as well as assess the alarm validity to accurately make a decision as to 
whether the alarm warranted interventions. Advanced beginner nurses responded, "To figure out what alarm it is, or where it is coming from [cardiac monitor or other equipment]". "I assess the patient, look at what is alarming, and see if I have enough information after I assess the patient". Responses from expert nurses including, "First I have to look at the alarm to see what it is, but you also have to look at the patient, that is the important thing", "Look at the patient, look and see what the alarm is and correlate the two", "If it's life threatening, even if it is not your patient, get up and go in there, and see what is going on".

Resetting Default Alarm Parameters. Decision to reset alarm parameters was based on providers' treatment orders, after observing the patient's baseline vital signs, with a change in patient's clinical condition, and nurses' comfort level. Resetting alarm parameters was done when nurses came on shift once they have assessed their patients. Eighty-six percent of nurses interviewed stated that they understood the need to set parameters specifically to their patients' baseline and $50 \%$ of expert nurses would observe the patient before adjusting the parameters. Responses from advanced beginners included: "It always depends on the patient. I try to figure out [the parameters] based on if the patient is asymptomatic and if this [parameters] is normal for the patient". "If the patient became unstable, like if the patient coded, you would just start from scratch because they are no longer at their baseline". Responses from expert nurses related to resetting monitor parameters included: "The only time I would do that [resetting the default alarm parameters] is once I know their [the patient's] baseline". "After watching the monitor for a couple of hours and seeing which alarms are going off more often and where my patient's normality is". "It depends on what is ok with the physician. If the physician is ok with the patient's [heart rate] running in the 130s... Maybe he [the patient] just needs more fluids. Maybe I'd up them [the parameters] until they [patient's vital signs] came back to normal". "When they [the parameters] are not appropriate for that particular patient, l'd like to customize monitor alarms based on my patient's [unique condition]". One expert nurse interviewed did not see the 
need to adjust monitor parameters and another one indicated that nurses were not allowed to adjust alarms unless given specific parameters from the physician. "Reset the parameters? I don't think we reset them, do we? I don't reset them."When inquiring about parameter settings that made the nurse uncomfortable, an advanced beginner responded: "It depends on the situation. I am actually pretty anxious. If it is normal for the patient or physician has specified otherwise, then it's okay to go out of the [default] parameters. if there is too big of a deviation [from patient's normal or default parameters], I will get a second and third opinion". An expert nurse responded: "Settings that would not allow me to intervene in a timely manner: a pressure that is too low, respiratory rates that are too low."

Frequent False Alarms Related to Blood Pressure (BP). All nurses felt BP readings needed to be accurate and would intervene to minimize false alarms often by troubleshooting the equipment or BP acquisition site. An advanced beginner responded: "Well, you need to figure out why it $[B P]$ is not true. If the A-line [arterial-line] is not patent, then you try to adjust it, fix it or pull it. You just can't have inaccurate values recorded in the medical record." An expert nurse responded: "I would try to troubleshoot the problem to see why I am getting the false alarms and reset my parameters on my monitor".

Frequent False Alarms Related to Heart Rate or Arrhythmia. For frequent false heart rate or arrhythmia alarms, nurses would determine if there is a problem with the monitoring system, a restless patient, or parameters not set appropriately for that patient. Thirteen percent of nurses indicated that they do not attend to these alarms, allow them to sound, or silence them. A nurse with 2.5 years of ICU experience stated, "Look at the patient, are they moving around? Check the placement of the leads and go from there." A nurse with 5 years of ICU experience stated," I wouldn't do anything, continue to monitor them [the alarms]". Responses from expert nurses included: "You can silence [the alarm], like if the patient is in A fib [atrial fibrillation]. You can turn off the yellow alarms, the irregular rate one". "Are the parameters right? Are the 
electrodes right? Are they [electrodes] in the right position?"

Frequent False Alarms Related to Pulse Oximetry. Nurses indicated that false pulse oximetry readings are frequent problems. Solutions included changing the probe sensor to a site with good perfusion, improve perfusion by warming the extremity, or changing the probe sensor, cable, or monitor module. An advanced beginner nurse responded, "I would try to keep moving it [oximetry probe sensor] on the patient until it [pulse oximetry] wasn't alarming and was reading what seems to be correct". Expert nurses also considered patient treatments that could interfere with pulse oximetry reading, such as vasopressor use. An expert nurse replied, "Pulse oximetry is kind of a nightmare in and of itself. you know- Is it a good sensor? Is it in the right place? Is it on the non-dominant arm? is it an old cuff? Are they [patients] on pressors [vasopressors]?

Decision on Disabling Alarms. Level of an alarm (e.g., crisis, advisory), presence of a backup warning mechanism, patients' clinical status, and the nurses' comfort level are factors taken into account while judging if an alarm may be disabled. For example, $62 \%$ of expert nurses would disable the apnea alarm on the cardiac monitor in a ventilated patient and use ventilator apnea alarm as the backup. However, $25 \%$ of nurses stated that they would never disable an alarm unless the patient was transitioning to step-down or comfort care. All nurses interviewed expressed that disabling alarms on patients who are placed on comfort care is an acceptable practice, though $60 \%$ of advanced beginner nurses were hesitant about disabling any alarm. All nurses indicated that alarms for BP, arterial line, and oxygen saturation should not be disabled at any time. An advanced beginner responded, "No, [I do not disable alarms] even when they keep going off...I am scared if the one time I disabled it [the alarm] something really bad is going to happen and I don't want that'. Expert nurses' responses related to disabling monitor alarms included: "I do, yes I do. I disable respiration alarms [on the cardiac monitor] if they [patients] are on the ventilator because there is no point to even having those 
[alarms]". "The only exception [I disabled the alarm] was a patient placed on comfort care because the alarms were irritating to the family and did not require intervention".

Decision on Seeking Consultation. Regardless of length of ICU experience, all nurses interviewed expressed that they do not hesitate to consult a colleague when the patient's safety is in question. When nurses are unsure if an alarm is real, they tended to err on the side of caution, perform additional assessment and obtain a second opinion. Advanced beginner nurses were more likely to call for help from another nurse or the charge nurse. Expert nurses tended to take more time to assess the situation before calling for help. Responses from advanced beginner nurses included, "When I need help, if the patient is getting sicker real fast and I cannot manage the patient alone", "If I am unsure of a certain course of action or if I come across something new, I try to get their [colleagues'] inputs. There are a lot of experienced nurses around here. I go to them". An expert nurse stated, "When I question my own ability to figure out what is going on, or if what I thought was going on was true but I did not know what to do about it". In addition, nurses' trust in a colleague's judgement is reliant on their previous experience with that person in a critical situation. They often solicited guidance from someone with whom they have had a positive working experience. "You learn who to trust by working with them and know their level of expertise. You also learn who not to trust".

\section{Evaluation}

Barriers to Response to Alarms. Difficulty in responding to alarms included low staffing, multiple job responsibilities, and competing priority tasks. Responses from advanced beginner nurses included: "Sometimes it is just impossible to manage alarms because we are low staffed. I can't even emphasize my frustration with that". "Call lights, multiple alarms going off at one time, and putting orders in. If you are putting in orders on a patient, you block everything out so you can focus." An expert nurse replied, "I have multiple jobs at one time. It is hard for me to physically go to two different areas to respond [to alarms]. When I am in charge, it is not only 
the alarms in the ICU I have to respond to but also to codes in the whole hospital. Plus, I am in the count. There is only a certain amount of alarms I can respond to."

Consequences of Disabling Alarms. All nurses interviewed were aware that disabling alarms in ICU patients can have dire consequences. An advanced beginner stated: "I am sure bad things have happened many times before. That is why disabling something [alarms] should not be done lightly... That's why continuity of care is important. Once you know a patient, you are a little more comfortable which alarms you can turn off and which you can't". An expert nurse responded, "Oh yea, absolutely. If you disable them [alarms] you are missing the whole point of an alarm. You can compromise patient safety if you disarm the alarm”.

Determination of Adequate Alarm Management. Nurses felt that adequate alarm management can be determined if the alarms sounded are valid, the number of false alarms are decreased, patients are safe, and patients do not complain about the constant noise and sleep interruption. An advanced beginner nurse responded, "I really judge it [adequacy of alarm management] off how safe I feel the patient is, and how well I feel they are doing. I feel like if the alarms keep going off, something is not right. I have to figure it out". Responses from expert nurses included," I have had patients tell me that they have gotten more sleep than they have in a while. They actually have not gotten sleep because of what is going on with their alarms", "Do I have many false alarms? Can I believe the ones that I have? ... because for me I should always believe my alarms until proven otherwise."

Training Received on Monitors. Only $25 \%$ of nurses $(n=4)$ stated that they were familiar with monitors used in the study ICU and among these, only $50 \%(n=2)$ had formal training. All nurses stated that they were shown the "basics" by their preceptors during orientation and this brief training was limited to the expertise and experience of their preceptors. "I didn't get any training on how to use the monitors. During my orientation it was - these are the monitors, here is where you silence them, that was about it". "When we first opened [this hospital], they trained 
us on [how to use] the monitors." "I had used these monitors a long time ago but basically you only get what your preceptor taught you". Several nurses also expressed that lack of formal training with monitors was also an issue in their prior employment.

\section{DISCUSSION}

Decision-making is "the process of reaching a judgement or choosing an option, sometimes called a course of action, to meet the needs of a given situation." ${ }^{20}$ Across different occupations, examples of the importance of decision-making skills for safe task performance are abundant; ${ }^{20}$ however, research on decision-making in healthcare during task execution especially under high pressure and uncertainty conditions is needed. Here we Investigated individual nurse's cognition, specifically decision-making related to device alarms, in real world contexts.

Proliferation of sophisticated physiological monitoring and treatment devices has become an integral part of intensive care. Nurses indicated that they felt overwhelmed by device alarms and these alarms are also disruptive to patients. Yet, the number of hourly alarms they heard or dealt with varied greatly within the same ICU with only $38 \%$ heard more than five alarms per hour in their last shift. Pervasive low urgency and false alarms can become ongoing background noise to the point that the staff is not fully aware of the sound. ${ }^{21}$ If the nurse experienced a history of false alarms with a particular device, the alarm may be ignored easily if it went off inappropriately. ${ }^{21}$ Funk, et al. ${ }^{22}$ infer that excessive alarm racket can become "white noise" that nurses ignore or rely on the monitor technician to inform them of important alarms. The low numbers of monitor alarms heard by some nurses in the present study may be due to nurses tuning-out white noise and only hearing alarms with high urgency or rely on the monitor technician to inform them of important alarms. A nurse with 20 years of ICU experience reasoned "The fact that we have a monitor technician now in the ICU I think most people really don't pay any attention [to the alarms], including myself unless it [the alarm] is specifically from 
my patient."

ICU nurses integrate multiple factors into considerations when deciding how quickly or even whether an alarm will be responded to. These included tone, duration, and type of the alarm, priority of other competing activities, adequacy of staffing, knowledge of patient acuity, and years of ICU experience. Nurses do not judge the urgency of an alarm in isolation. They integrated knowledge of the patient's condition and immediate visualization of the patient into their judgement. The nurses felt that the ability to make such judgment is enhanced by knowledge of the monitoring system and situational experience.

$\underline{\text { Tone, Duration, and Type of Alarm }}$

Audible alarms can improve patient safety when they are employed properly in the work environment. Factors that affect nurses' promptness to respond to the alarm often depends on the volume, duration and type of the alarm sound. Thirteen percent of nurses indicated that they do not attend to heart rate and arrhythmia alarms, allow them to sound, or silence them, likely due to high frequency of false alarms.

Audible alarms, like the ones embedded in the medical devices, are considered abstract alarms. Connection of these abstract alarms with urgency is learned when the operator (nurse) knows what the alarm means and reacts accordingly. ${ }^{23}$ Cropp and colleagues tested whether ICU staff could quickly recognize life-threatening alarms better than the less urgent ones, by sound alone, and reported that critical alarms were correctly identified only half the time and noncritical sounds only 40 percent of the time by ICU personnel. ${ }^{24}$ Nurses with more than 1 year of experience in the ICU could recognize better noncritical sounds but not the critical ones. Cropp et al. concluded that the myriad of alarms regularly occur in the ICU are too much for even experienced ICU staff to quickly discern. ${ }^{24}$ Trainings to include a full range of alerting sounds that ICU nurses are expected to encounter would be beneficial in the ICU setting. Competing Activities/Staffing 
Low staffing, multiple job responsibilities, and competing priority tasks are barriers for ICU nurses to respond to alarms. When the nurse is genuinely too busy on tasks of equal or greater importance, it is not surprising that they are unable to respond to the new alarm. In the present study, one third of advanced beginner nurses indicated that they might ignore the alarm when they are engaged in a higher priority task. This could be a result of generally inadequate staffing or an excess of simultaneous or near simultaneous events. ${ }^{21}$ When workload gets high and there are too many competing demands, adequate staffing and workload evaluation should be part of effective alarm management strategies.

\section{$\underline{\text { ICU Experience }}$}

All nurses felt that they need to validate the alarm by correlating their visualization of the patient and the types of alarms. When nurses are unsure if an alarm is real, they tended to err on the side of caution. ICU nurses with less experience tend to appropriately consult a colleague sooner while nurses with more experience tend to perform additional assessment before calling for help. The first priority on management of false alarms was to assure the patient is safe before trouble-shooting the equipment, including changing electrocardiographic electrodes and pulse oximeter probes or probe sites.

Less experienced nurses tend to rely on monitor alarms to alert them to a patient problem and are more tolerant of alarms sounding due to a lower threshold of comfort with re-setting or silencing alarms. Their hesitancy to disable any alarm likely due to lack the exposure to repeat patterns that enhance their judgment capability. ${ }^{25}$ Novice nurses' decision-making characteristically tends toward rule-based thinking, with a focus on task completion or responding to discrete patient issues, thus more likely to ignore an alarm if they are engaged in a task that is deemed higher in priority.

Expert nurses tend to integrate patterns and factual informational cues with situational experience to inform their decisions ${ }^{25}$ and are more likely to view patient situations as a whole 
and within context. ${ }^{26}$ It has also been reported that expert nurses make a judgment more quickly in a critical clinical situation without having to waste time considering options that are not helpful. ${ }^{25}$ In this case, nurses with extensive ICU experience tend to correlate tone and duration of alarm with knowledge of patient condition to guide their response to an alarm and consider extensive clinical variables when troubleshooting false alarms (e.g. vasopressor diminishing perfusion which interferes pulse oximetry readings). In addition, expert nurses are more comfortable than novice nurses to re-set alarm parameters and such practice is often done after nurses have the chance to assess their patients and their individualized baselines after assumed care.

\section{Inadequate Training on Alarm Devices}

Data from the present study revealed a very concerning practice issue - the majority of ICU nurses indicated that they were unfamiliar with the cardiac monitors used and had little or no formal training with the cardiac monitors. The brief training received by critical care nurses was limited to the expertise and experience of their preceptors, a common theme emerged as "these are the monitors, here is where you silence them...". This finding is consistent with prior report in that critical care nurses attribute their frustration to alarms due to poor usability of the complex alarm devices, specifically the cardiopulmonary monitoring systems. ${ }^{27,28}$ Nurses reported that newer cardiac monitors to be "very complex and not user friendly" and it takes a long time for one to feel comfortable interacting with physiologic monitors. ${ }^{27}$

"Inadequate staff training on the proper use and functioning of (alarm-related) equipment" has been recognized by the Joint Commission. ${ }^{29}$ Nurses interviewed in the present study felt a formal training would strengthen their ability to manage alarms. However, traditional group-based one-time training on complex alarm-equipped monitoring devices has been reported to be inadequate to decrease alarm burden. ${ }^{30}$ Future studies on alarm management should focus on effective use of alarm-related equipment trainings and/or 
innovative user-centered alarm management clinical decision support systems to enhance intuitive nurse-device interactions.

\section{Study Limitations}

A limitation of this study is the small sample of nurses from a single ICU. Participants from multiple ICUs would enrich the findings. In addition, Benner's definition of advanced beginner was a nurse who has had prior experience in actual clinical situations and the competent nurse has had 2-3 years of experience in the same job situation and is able to demonstrate ability and confidence in their decisions. ${ }^{19}$ Due to the small sample size, advanced beginners and competent nurses were combined as "advanced beginners." Though some differences have emerged in phases of clinical decision-making process related to alarm management between advanced beginners and expert nurses, it is likely that arbitrary years of clinical experience may not fully reflect competency.

\section{CONCLUSION}

Noise pollution caused by excessive monitor alarms and the hazards of alarm fatigue is well known. This study uniquely gained insight into the intricacy of the ICU nurses' critical decision process when responding to device alarms using the Situated Clinical Decision-Making Framework, namely constructs of cues, judgment, decision, and evaluation, with a semistructured interview. We found that ICU nurses integrate multiple subjective and objective factors to decide their responses to monitor alarms and their management. Nurses with less years of experience are more tolerant of alarms sounding due to a lower threshold of comfort with re-setting or silencing alarms while ICU nurses with more extensive experience are more comfortable re-setting alarm limits to the patient's baseline. Understanding the decision-making process used by nurses can guide the development of policies and learning experiences that are crucial clinical support for alarm management. Optimizing nursing orientation to include structured training with monitors can improve alarm management, decrease the number of false 
alarms, decrease alarm fatigue, and ultimately improve patient safety and patient outcomes. 


\section{REFERENCES}

1. Ebright PR, Patterson ES, Chalko BA, Render ML. Understanding the complexity of registered nurse work in acute care settings. J Nurs Adm. 2003;33(12):630-638.

2. Donchin $\mathrm{Y}$, Seagull FJ. The hostile environment of the intensive care unit. Current opinion in critical care. 2002;8(4):316-320.

3. Drew BJ, Harris P, Zegre-Hemsey JK, et al. Insights into the problem of alarm fatigue with physiologic monitor devices: a comprehensive observational study of consecutive intensive care unit patients. PloS one. 2014;9(10):e110274.

4. Atzema C, Schull MJ, Borgundvaag B, Slaughter GR, Lee CK. ALARMED: adverse events in low-risk patients with chest pain receiving continuous electrocardiographic monitoring in the emergency department. A pilot study. Am J Emerg Med. 2006;24(1):62-67.

5. Lawless ST. Crying wolf: false alarms in a pediatric intensive care unit. Crit Care Med. 1994;22(6):981-985.

6. Tsien CL, Fackler JC. Poor prognosis for existing monitors in the intensive care unit. Crit Care Med. 1997;25(4):614-619.

7. Chambrin MC, Ravaux P, Calvelo-Aros D, Jaborska A, Chopin C, Boniface B. Multicentric study of monitoring alarms in the adult intensive care unit (ICU): a descriptive analysis. Intensive care medicine. 1999;25(12):1360-1366.

8. Imhoff M, Kuhls S, Gather U, Fried R. Smart alarms from medical devices in the OR and ICU. Best Pract Res Clin Anaesthesiol. 2009;23(1):39-50.

9. O'Carroll TM. Survey of alarms in an intensive therapy unit. Anaesthesia. 1986;41(7):742-744.

10. Talley LB, Hooper J, Jacobs B, et al. Cardiopulmonary monitors and clinically significant events in critically ill children. Biomedical instrumentation \& technology / Association for the Advancement of Medical Instrumentation. 2011;Suppl:38-45.

11. Weil KM. Alarming monitor problems. Nursing. 2009;39(9):58.

12. Gorges M, Markewitz BA, Westenskow DR. Improving alarm performance in the medical intensive care unit using delays and clinical context. Anesth Analg. 2009;108(5):15461552.

13. Militello LG, Hutton RJ. Applied cognitive task analysis (ACTA): a practitioner's toolkit for understanding cognitive task demands. Ergonomics. 1998;41(11):1618-1641.

14. International Ergonomics Association. http://www.iea.cc/whats/index.html. Accessed December 8, 2017.

15. Hassall M, Xiao T. Human Factors and Ergonomics. In: Wright JD, ed. International Encyclopedia of the Social and Behavioral Sciences. Vol 8. 2nd ed. Amsterdam, Netherlands: Elsevier; 2015:297-305.

16. Flin R, Winter J, Cakil Sarac, Raduma M. Human factors in patient safety: Review of topics and tools. World Health Organization;2009.

17. Gillespie M. Using the Situated Clinical Decision-Making framework to guide analysis of nurses' clinical decision-making. Nurse education in practice. 2010;10(6):333-340.

18. Gillespie M, Peterson BL. Helping novice nurses make effective clinical decisions: the situated clinical decision-making framework. Nurs Educ Perspect. 2009;30(3):164-170. 
19. Benner P. From novice to expert. The American journal of nursing. 1982;82(3):402-407.

20. Flin RH, O'Connor P, Crichton M. Safety at the sharp end: A guide to non-technical skills. Boca Raton, FL CRC Press 2008.

21. Hyman WA, Johnson E. Fault tree analysis of clinical alarms. Journal of Clinical Engineering. 2008(April/June):85-94.

22. Funk M, Clark JT, Bauld TJ, Ott JC, Coss P. Attitudes and practices related to clinical alarms. American journal of critical care : an official publication, American Association of Critical-Care Nurses. 2014;23(3):e9-e18.

23. Guillaume A. Intelligent auditory alarms. In: Hermann T, Hunt, A., Neuhoff, J. G.,, ed. The Sonification Handbook. Berlin, Germany: Logos Publishing House; 2011:493-508.

24. Cropp AJ, Woods LA, Raney D, Bredle DL. Name that tone. The proliferation of alarms in the intensive care unit. Chest. 1994;105(4):1217-1220.

25. Crandall B, Klein G, Hoffman R. Working Minds: A practitioner's guide to cognitive task analysis. Cambridge, MA: The MIT Press; 2006.

26. Benner PE, Tanner CA, Chesla CA. Expertise in nursing practice: Caring, clinical judgment, and ethics. New York, NY: Springer Publishing Company; 1996.

27. Sowan AK, Tarriela AF, Gomez TM, Reed CC, Rapp KM. Nurses' Perceptions and Practices Toward Clinical Alarms in a Transplant Cardiac Intensive Care Unit: Exploring Key Issues Leading to Alarm Fatigue. JMIR human factors. 2015;2(1):e3.

28. Drews F. Patient Monitors in Critical Care: Lessons for Improvement. In: Henriksen K BJ, Keyes MA, Grady ML, ed. Advances in Patient Safety: New Directions and Alternative Approaches (Vol. 3: Performance and Tools). Rockville (MD): Agency for Healthcare Research and Quality (US); 2008:1-13.

29. Alert SE. New Alert Promotes Medical Device Alarm Safety in Hospitals. Joint Commission Perspectives. 2013.

30. Sowan AK, Gomez TM, Tarriela AF, Reed CC, Paper BM. Changes in Default Alarm Settings and Standard In-Service are Insufficient to Improve Alarm Fatigue in an Intensive Care Unit: A Pilot Project. JMIR human factors. 2016;3(1):e1. 